\title{
82. LOCAL MAGNETIC FIELD AND ITS RELATIONS TO THE LOCAL SPIRAL ARM STRUCTURE
}

\author{
G. D. VER SCHUUR \\ National Radio Astronomy Observatory, Charlottesville, Va., U.S.A.
}

No paper was submitted. 